Nephrologe 2018 $\cdot 13: 222-223$

https://doi.org/10.1007/s11560-018-0263-1

(c) Springer Medizin Verlag GmbH, ein Teil von Springer Nature 2018

CrossMark

\author{
M. Zeier' ${ }^{1}$ T. Feldkamp ${ }^{2}$ \\ 'Sektion Nephrologie, Universitätsklinikum Heidelberg, Heidelberg, Deutschland \\ ${ }^{2}$ Klinik für Innere Medizin IV, Nieren- und Hochdruckkrankheiten, Universitätsklinikum Schleswig- \\ Holstein, Christian-Albrechts-Universität Kiel, Kiel, Deutschland
}

\title{
Bildgebung in der Nephrologie
}

Die Diagnostik in der Nephrologie ist auf die Bildgebung der Nieren und des ableitenden Harnsystems angewiesen. Die Diagnostik umfasst sowohl die Nierenmorphologie (Größe), die Durchblutung (Duplexverfahren) als auch die onkologische Diagnostik. Insbesondere die onkologische Diagnostik ist für den Nephrologen bedeutsam, da viele Patienten im Rahmen von Nierenfunktionsstörungen auch bildgebend untersucht werden müssen und damit auch als Zufallsbefund ein Nierenzellkarzinom entdeckt wird. Neben diesen Untersuchungsverfahren ist auch die Beurteilung des Harntransports wichtig. Zuletzt ist die bildgebende Diagnostik v. a. auch in der Pädiatrie in den letzten Jahren zunehmend wichtiger geworden und hat auch durch Ultraschallmethoden und Kernspintomographie erhebliche Innovationen erlebt.

PD Dr. Moritz vom Universitätsklinikum Schleswig-Holstein stellt die speziellen Fragestellungen der pädiatrischen bildgebenden Diagnostik vor. Sie nimmt bei dieser Patientengruppe einen großen Raum ein. In seiner Übersichtsarbeit werden v.a. die strahlenfreien Verfahren wie Ultraschall und Magnetresonanztomographie (MRT) ausführlich besprochen.

Die MRT-Bildgebung in der allgemeinen Nephrologie wird ausführlich von Prof. Dr. Hallscheidt behandelt. Hier geht es insbesondere um die Diagnostik von Nierentumoren, aber auch um die Beurteilung von z.B. Lebendspendern vor einer möglichen Nierenentnahme.

Ein weiterer wichtiger Punkt ist die Differenzierung zwischen auffälliger Zyste und einem tatsächlich nachweisbaren Nierenzellkarzinom. Einen klaren Algorithmus zum Management dieser zystischen Nierenläsionen stellt PD Dr. Stock in seinem Beitrag über die nephrologi- sche Ultraschalldiagnostik vor. Zusätzlich beschreibt er, wie die Ultraschalldiagnostik effektiv zur Beantwortung spezifischer nephrologischer Fragestellungen eingesetzt werden kann und welche technischen Möglichkeiten, wie z. B. der Einsatz von Taschenultraschallgeräten, die Ultraschalldiagnostik weiter verbessern.

\section{》) Es ist wichtig zu wissen, welche bildgebende Methode für welche Fragestellung am besten geeignet ist}

Eine wichtige Frage, die ebenfalls diskutiert wird, ist die der Notwendigkeit der Nuklearmedizin in der nephrologischen Diagnostik. Sie spielt in der Evaluation verschiedener Nierenerkrankungen eine wichtige Rolle, z. B. beim postrenalen Nierenversagen im Sinne von Harntransportstörungen. Zusätzlich liefert die Szintigraphie auch eine objektivierbare Nierenfunktionsleistung, die unter Umständen aus anderen Gründen nicht zu erheben ist. Neuere Verfahren wie z. B. die ${ }^{99 \mathrm{~m} T c-D M S A}$ liefern sogar eine sehr genaue Bestimmung der glomerulären Filtrationsrate. Dr. Rathke referiert in seinem Beitrag ausführlich über die Möglichkeiten der nuklearmedizinischen Diagnostik und stellt die Möglichkeiten für die Nephrologie dar.

Prof. Dr. Kribben und seine Mitarbeiter beschäftigen sich mit dem Kontrastmitteleinsatz bei bildgebender Diagnostik der Niere. In dieser sehr umfassenden Darstellung wird v. a. aufgezeigt, dass das kontrastmittelinduzierte Risiko des Nierenversagens lange Zeit überschätzt wurde. Dieser Sachverhalt ist besonders deshalb wichtig, da bei genau- 
er Indikationsstellung viele niereninsuffiziente Patienten von diesen kontrastmittelverstärkten bildgebenden Verfahren profitieren. Eine nicht ausreichende Bildgebung wegen eingeschränkter Nierenfunktion kann durchaus zur Unterdiagnostik und damit zu fehlgeleiteter Therapie dieser Patientengruppe führen.

Zusammenfassend braucht die $\mathrm{Ne}$ phrologie die bildgebende Diagnostik auf den verschiedensten Ebenen. Es ist wichtig $\mathrm{zu}$ wissen, welche Methoden für welche Fragestellungen am besten geeignet sind bzw. wie sie sich am besten ergänzen. Dabei sind verschiedene Aspekte zu beachten, z.B. in der Pädiatrie der Strahlenschutz und in der Altersmedizin insbesondere die leichtbis mäßiggradig eingeschränkte Nierenfunktion.

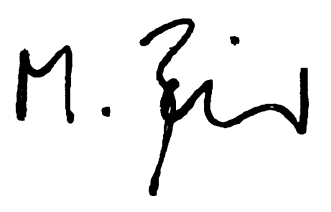

Prof. Dr. Martin Zeier

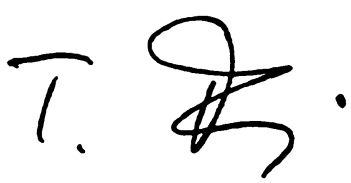

Prof. Dr. Thorsten Feldkamp

\section{Korrespondenzadresse}

Prof. Dr. M.Zeier
Sektion Nephrologie,
Universitätsklinikum
Heidelberg
Im Neuenheimer Feld 162,
69120 Heidelberg,
Deutschland
martin.zeier@med.uni-
heidelberg.de

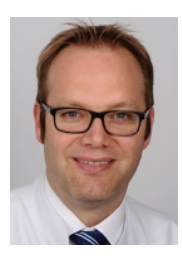

Prof. Dr. T. Feldkamp

Klinik für Innere Medizin IV, Nieren- und Hochdruckkrankheiten, Universitätsklinikum Schleswig-Holstein, ChristianAlbrechts-Universität Kiel Rosalind-Franklin-Str. 12, 24105 Kiel, Deutschland thorsten.feldkamp@uksh.de

Interessenkonflikt. M. Zeier und T. Feldkamp geben an, dass kein Interessenkonflikt besteht.

\section{Genetischer Auslöser für congenitales mesoblastisches Nephrom entdeckt}

Das congenitale mesoblastische Nephrom (CMN) kann bereits in den ersten Lebensmonaten von Säuglingen oder sogar schon vor der Geburt auftreten. Glücklicherweise ist der Nierentumor sehr selten und lässt sich oftmals mit einem chirurgischen Eingriff heilen. Weitere spezifische Behandlungsmöglichkeiten existieren jedoch nicht - auch wegen der bislang ungeklärten Ursachen dieses Tumors. Drei Unterarten dieses Nierentumors sind bekannt: das klassische, das zelluläre und das gemischte Nephrom. Für das zelluläre CMN ist seit Langem eine charakteristische Veränderung der Chromosomen als Auslöser bekannt. In diesem Fall fusionieren zwei Gene miteinander, was zu einer überschießenden Aktivität eines Enzyms führt. Für die klassische Variante des CMN waren bislang keine typischen genetischen Veränderungen bekannt. Für das klassische CMN hat ein internationales Forscherteam jetzt erstmals einen genetischen Auslöser identifiziert. Die Ergebnisse werden in der aktuellen Ausgabe der Fachzeitschrift Nature Communications vorgestellt.

Durch Genomanalysen von Tumor- und Blutproben der Patienten konnte in gut $70 \%$ der klassischen CMN eine neuartige Mutation des Rezeptors für den epidermalen Wachstumsfaktor (EGFR) nachgewiesen werden. Die Wissenschaftler entdeckten im Erbgut der betroffenen Zellen eine Verdoppelung der enzymatisch aktiven Kinase-Region. Dies hat zur Folge, dass der EGF-Rezeptor überaktiv wird und die Tumorzellen dauerhaft zum Wachstum angeregt. Wie weitere Untersuchungen zeigten, weist auch die gemischte Variante des Tumors in der Mehrzahl der Fälle eine solche Mutation auf.

Sowohl bei der klassischen als auch bei der zellulären Variante des CMN starten die genetischen Veränderungen einen der wichtigsten Signalwege für die Aktivierung des Zellwachstums: die MAP-Kinase-

Kaskade. Dabei schalten sie unter anderem die in der Zelle vorliegende BRAF-Kinase an. Das Gen für dieses Protein war auch in einigen der Tumoren mutiert, in denen weder die für die klassische, noch die für die zelluläre Variante verantwortliche Mutation nachgewiesen werden konnten. In diesen
Fällen kam es im betroffenen Gen zum Verlust einer Region, die für die Hemmung der BRAF-Kinaseaktivität zuständig ist, mit der Folge, dass das Protein daueraktiv ist und die MAP-Kinase-Kaskade angeschaltet bleibt.

\section{Parallelen zu Tumoren bei Erwachsenen}

Dem Forscherteam ist es damit gelungen, diesen seltenen Tumor des Säuglingsalters in nahezu allen Fällen auf die Aktivierung eines zentralen Signalweges zurückzuführen, der auch in vielen Tumoren des Erwachsenenalters eine wesentliche Rolle spielt. Insbesondere für CMN-Patienten, die chirurgisch nicht ausreichend behandelt werden können, liefern diese Erkenntnisse jetzt möglicherweise neue Behandlungsansätze durch die Übertragung bewährter Therapieprinzipien der Erwachsenenonkologie auf die Therapie des congenitalen mesoblastischen Nephroms.

Literatur: Wegert J., Vokuhl C. et al (2018) Recurrent intragenic rearrangements of EGFR and BRAF in soft tissue tumors of infants. Nature Communications, doi: 10.1038/s41467-018-04650-6 